\title{
El desarrollo social en el proceso de formación profesional desde la Química Orgánica
}

\section{The social development inside the process of profesional formation from Organic Chemestry}

\author{
MSc. Lázara Puerta Díaz ${ }^{1}$ \\ Ipuerta@ucf.edu.cu \\ MSc. Betsy Álvarez Vega² \\ balvarez@ucf.edu.cu
}

MSc. Yissel Pérez de Villa Amil Sellés ${ }^{3}$

ypvilla@ucf.edu.cu

Recibido: 11/12/2019; Aceptado: 4/3/2020

\begin{abstract}
RESUMEN
Las transformaciones en el contexto universitario forman parte del proceso educativo para desarrollar al máximo las potencialidades de los individuos. En correspondencia con el modelo del profesional este trabajo le confiere importancia a la formación integral y la cultura científica necesaria para el desarrollo social. Se exponen los resultados de la aplicación de la estrategia curricular del Programa Director de Promoción y educación para la salud y la sexualidad en el PEA de la Química Orgánica II. Se presentan actividades docentes relacionadas con los aminoácidos y su relevancia en el cambio oportuno de actitudes y la prevención de enfermedades como aspecto de interés político de nuestra sociedad. Se asumen los intereses colectivos e individuales de los estudiantes. Este tema sirve de modelo para el resto de los contenidos del programa. Se aplicó en el grupo de 4to año de la carrera Licenciatura en educación especialidad Química en el curso 2019-2020 en la Universidad de Cienfuegos Carlos Rafael Rodríguez.
\end{abstract}

Palabras clave: Aminoácidos, desarrollo social

\footnotetext{
${ }^{1}$ Docente de la Universidad Carlos Rafael Rodríguez, Cienfuegos, Cuba.

2 Docente de la Universidad Carlos Rafael Rodríguez, Cienfuegos, Cuba.

${ }^{3}$ Docente de la Universidad Carlos Rafael Rodríguez, Cienfuegos, Cuba.
} 


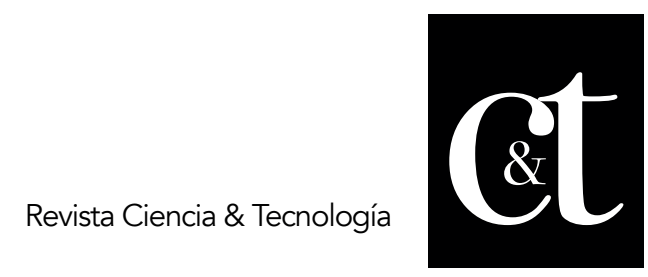

No. 26, 30 de abril de 2020

ISSN impreso: 1390 - 6321

ISSN online: 2661 - 6734

\begin{abstract}
The transformations in the university context are part of the educational process to maximize the potential of individuals. In correspondence with the professional model, this work gives importance to the integral formation and scientific culture necessary for social development. The results of the application of the curricular strategy of the Program Director of Promotion and education for health and sexuality in the PEA of Organic Chemistry II are presented. Teaching activities related to amino acids and their relevance in the timely change of attitudes and the prevention of diseases as an aspect of political interest of our society are presented. The collective and individual interests of the students are assumed. This theme serves as a model for the rest of the program contents. It was applied in the group of 4th year of the degree in specialty chemistry education in the 2019-2020 course at the University of Cienfuegos Carlos Rafael Rodríguez.
\end{abstract}

Keywords: Amino acid, social development

\title{
Introducción
}

La Educación es un aspecto necesario en el desarrollo de cualquier sociedad. Sus escenarios son significativos para elevar la calidad de vida de los sujetos mediante la adquisición de conocimientos y el desarrollo de hábitos y habilidades, así como la formación de valores. La prevención de enfermedades puede tener su influencia desde la contextualización de la educación y permite considerar disimiles situaciones del orden económico, político y social. Desde esta perspectiva, el sector se privilegia para transformar la actividad en el intercambio de análisis, reflexión y divulgación de experiencias de carácter científico. Así el proceso formativo se convierte en el espacio de preparación y creatividad de los estudiantes y docentes.

Siendo uno de objetivos de la educación cubana, el crecimiento personal, se empeñan los esfuerzos en la formación de profesionales acordes a las exigencias de calidad de la educación superior. Por ello, desde el Ministerio de Educación se orienta incluir las estrategias curriculares en los Programas de las distintas asignaturas y promover la calidad del egresado donde se exige la preparación constante del personal docente. Otras alternativas en el proceso de formación profesional lo constituyen los cursos optativos y propios que son considerados necesarios en el plan de estudio porque prevé las necesidades de formación y contribuir mediante el PEA al bienestar social de todos y la formación ciudadana como principios esenciales en estos procesos formativos.

Son varios los autores como Álvarez (2007), Delgado (2009), Addine (2004), Rico (2003), Carvajal (2000), que enfatizan en la educación contextualizada y transformadora. En este sentido, la preparación científica adquiere gran importancia al favorecer cambios en las actitudes de los sujetos si se mantiene la sistematicidad y la actualización de la ciencia donde el sujeto se alfabetiza y promueve la participación activa en los asuntos de la ciencia con la ética necesaria ante los grandes avances y desafíos sociales. Asimismo, se informa en diferentes asignaturas los programas de salud que el Estado ha puesto a la disposición del pueblo para considerar la calidad de vida mediante la prevención de enfermedades como los resultados de gran relevancia que ha tenido la ingeniería genética.

En el grupo de 4to año de la carrera de licenciatura en educación especialidad Química, al desarrollar el PEA de la Química Orgánica que se basa en el estudio de las sustancias al establecer las relaciones entre la estructura-propiedades- aplicaciones, se constató que el $100 \%$ de los estudiantes carecían de elementos fundamentales del conocimiento 


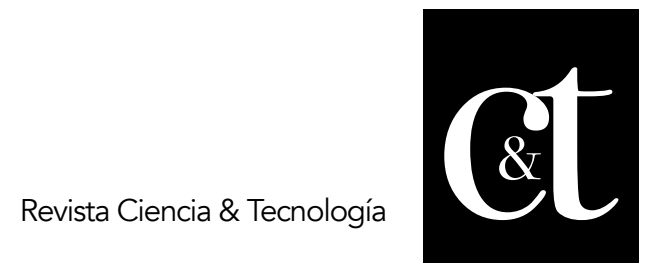

No. 26, 30 de abril de 2020

ISSN impreso: 1390 - 6321

ISSN online: 2661 - 6734

y el desarrollo de hábitos y habilidades al vincular los contenidos con la educación para la salud. Esta situación incide en la preparación cultural para enfrentar su desarrollo personal y también influye en los procesos educativos de los estudiantes que forman como parte de su actividad laboral. El $50 \%$ de los estudiantes mencionan que los compuestos orgánicos ejercen funciones en el organismo humano, pero no dominan las características esenciales de las mismas.

Se constató que desde la preparación de la disciplina se orienta la actualización científica para impartir las asignaturas, pero no se hace énfasis en como relacionar los contenidos con el Programa de promoción y educación para la salud y la sexualidad (PDPESS). De ahí, que los análisis de la situación anterior condujeron a que el trabajo del colectivo de disciplina dirigiera su atención a elevar la cultura científica mediante la aplicación del programa director de salud con énfasis en la prevención de enfermedades y alcanzar índices superiores de calidad en la formación profesional desde el PEA de la Química orgánica.

\section{Desarrollo}

Las Universidades en Cuba tienen el encargo social de la formación profesional y este se concreta en el trabajo personalizado con el estudiante. En este proceso formativo intervienen los docentes del colectivo de carrera y las personas que están vinculadas al estudiante desde el entorno social y laboral. Las exigencias que ello ocupa se dirigen a un objetivo fundamental que es fomentar actitudes en los individuos acordes a un comportamiento social que conlleve al bienestar individual y colectivo.

Los fundamentos pedagógicos y didácticos del proceso educativo tienden a potenciar la educación científica mediante la formación integral de los individuos. En este interés se orientan hacia todos los niveles de enseñanza las transformaciones necesarias para la adquisición de conocimientos y el desarrollo de hábitos y habilidades. Son diversas las metodologías que proponen autores mediante la adquisición de conocimientos científicos, el desarrollo de habilidades y hábitos que le permitan a los individuos estar al nivel de su tiempo, o sea que se sientan aptos y capacitados para enfrentar el vertiginoso desarrollo de la ciencia y la técnica y poseer la ética de proteger el medio ambiente y comprometer sus acciones hacia el desarrollo sostenible, lo cual se convierte en patrones axiológicos.

De ahí que autores como Delgado (2009) y Álvarez (2007) destacan la importancia de la alfabetización científica de los individuos para ser capaces de analizar los avances científicos-tecnológicos y participar activamente en la solución de los asuntos de esta índole al considerar su impacto social. Rodríguez (2009), dirige su atención a la educación bioética.

Por otra parte, se resalta el papel del decente al cumplir con calidad los objetivos de la educación y la formación integral de cada estudiante con profundas convicciones, conocimientos sólidos de amor y conciencia de compromiso con la sociedad en que vive. Addine (2004) comprende la práctica social como realidad educativa y Carvajal (2000) con propuestas renovadoras respecto a una vida sana y responsable.

También la vinculación del conocimiento científico escolar con los conocimientos y experiencias de la vida diaria de los estudiantes y con su hacer práctico, contribuye, por un lado, a que dicho conocimiento sea más significativo y más apto para ser utilizado luego en diversas situaciones $y$, por ende, el aprendizaje de las ciencias adquiere mayor 


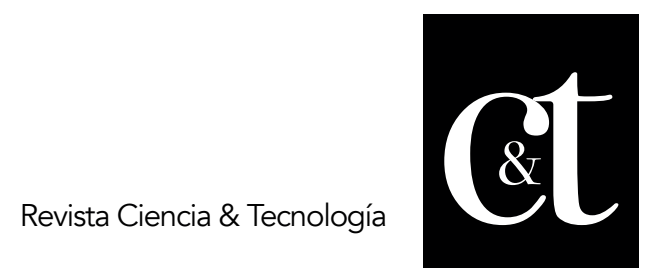

No. 26, 30 de abril de 2020

ISSN impreso: 1390 - 6321

ISSN online: 2661 - 6734

sentido y relevancia para ellos. Lo anterior posibilita la constante dinámica del PEA en las Universidades y reevaluar la naturaleza social de la ciencia al considerar los intereses económicos y políticos que la sustentan en nuestra sociedad.

Al respecto de los estudios CTS, se promueve políticas para generar los conocimientos en las prácticas sociales y estas a su vez intervienen en los procesos de formación ciudadana. Tanto en las vías de formación del pregrado y posgrado este objetivo es una prioridad en la Educación Superior. Un tema importante que adquiere relevancia en este trabajo es la calidad de vida de los sujetos en una sociedad por lo que repercute en la propia inserción del sujeto en el ámbito familiar y social. Según Carvajal (2000), considera la importancia de la educación para la Salud en el contexto educativo y la promoción desde los distintos niveles de enseñanza. Respecto a la educación superior se orienta que los estudiantes deben dominar los conocimientos y habilidades generales que les permitan una actuación consecuente y responsable en aspectos relacionados con la sexualidad, hábitos alimentarios, conductas higiénicas y prevención de enfermedades, tanto en su desarrollo personal como en su influencia educativa en los alumnos que forma.

En el PDPESS (2007), se deja espacio para que el docente ponga en acción sus potencialidades didácticas y creativas, en función de educar en salud y fomentar estilos de vida más sanos a partir del currículo. Respecto al eje temático Higiene personal y colectiva este comprende entre sus contenidos régimen de vida y enfermedades transmisibles y no transmisibles, que serán abordados en el PEA de la Química orgánica por ser una prioridad en Cuba la educación para la salud y ocupar espacios por distintas organizaciones como la Organización de las Naciones Unidas para la Educación, la Ciencia y la Cultura (UNESCO), el Fondo de Naciones Unidas para la Infancia (UNICEF), la OMS, el Consejo de Europa (CE) y la Comisión de la Unión Europea (CUE).

El régimen de vida, así como las enfermedades de los sujetos tiene incidencias en aspectos económicos, políticos y sociales. Por ello, la educación es un proceso de atención permanente para los gobiernos y la valoración de la influencia de los adelantos científicos contribuyen a la adquisición de nuevos estilos de vida que facilitan conductas adecuadas y asumir con responsabilidad estilos de vida sanos.

De ahí, que se revele lo significativo del proceso educativo en el desarrollo social. Desde esta perspectiva teórica, se realizó el estudio en la práctica educativa en la carrera Licenciatura en educación especialidad Química, en el 4to. año del curso por encuentro mediante la asignatura Química orgánica. Lo cual evidenció la necesidad de potenciar en los estudiantes el interés por la ciencia desde la perspectiva de las experiencias previas de los conocimientos científicos y reflexionar sobre aquellos aspectos que aun adolecen en su preparación desde el PEA, el vínculo con la vida diaria y su importancia para tener actitudes responsables y una vida sana. Desde esta perspectiva se resume en el siguiente gráfico, la intención de los autores para potenciar la formación profesional. 


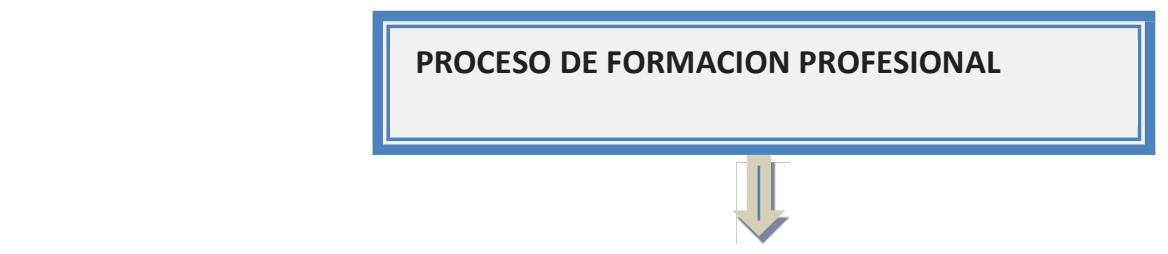

\begin{tabular}{|l||l|||}
\hline Modelo del profesional & Educación contextualizada \\
\hline
\end{tabular}

\section{Gráfico 1. Proceso de formación profesional y su vínculo con el desarrollo social desde el PEA de la Química orgánica \\ Fuente: Elaboración propia}

Se presentan actividades docentes para contribuir al eje temático higiene personal y colectiva mediante los contenidos régimen de vida y prevención de enfermedades al valorar aspectos de sanidad planteados en el PDPESS desde el PEA de la Química orgánica. Para ello, se asumen los criterios de grupos de especialistas del Ministerio de Educación (1988) al plantear que la actividad docente requiere para su realización en las escuelas o instituciones una correcta organización y dirección de la actividad. Además de tener un contenido previamente determinado en los planes de estudio y programas establecidos para cada uno de los niveles educacional.

Su principal objetivo es la asimilación de conocimientos científicos y lograr la formación de hábito, habilidades, y adquisición de procedimientos que le permita al individuo posteriormente orientarse en la sociedad. La actividad docente posee su propia estructura, que si bien coincide con los momentos fundamentales de cualquier actividad cognoscitiva (actividad constructiva, el juego, el trabajo), consta de tres momentos fundamentales: orientación, ejecución, y el control. Estos se basan en:

Orientación: radica en lograr de modo consciente y dirigido a los estudiantes, una orientación sobre las formas de solucionar problemas relacionados con el conocer y aprender, así como captar el procedimiento general que permite solucionar los problemas.

Ejecución: en esta etapa lo esencial es que los estudiantes adquieran los conocimientos básicos necesarios y asimilen los métodos y procedimientos para la obtención de los conocimientos por sí mismo. La asimilación de estos procedimientos determina la formación y desarrollo de habilidades comunes a todas las asignaturas y las especificas relacionada con determinados contenidos de algunas de ellas. Además, favorece el desarrollo intelectual de los estudiantes.

Control: resulta lo más importante por la contribución a la asimilación correcta de las acciones ante los estudiantes por la cual se llega a los resultados. En este el alumno aprecia sus insuficiencias, trabaja para su eliminación y toma consciencia de acercar sus resultados al nivel de las exigencias establecidas siendo así capaz de auto valorarse, y determinar en qué dirección y en qué medida debe empeñarse más.

En general, la orientación debe preceder a la ejecución y el control se realiza tanto en 


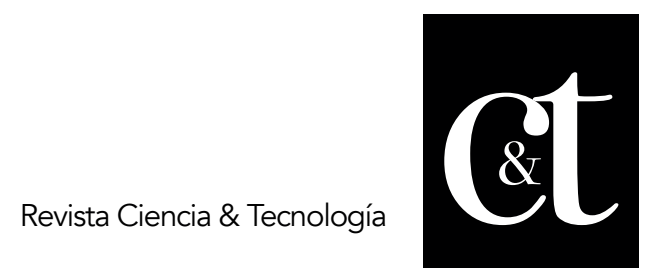

No. 26, 30 de abril de 2020

ISSN impreso: 1390 - 6321

ISSN online: 2661 - 6734

la orientación como en la ejecución.

Las actividades docentes que se presentan se caracterizan por ser flexibles y participativas. En ambos casos se consideran las condiciones reales del diagnóstico de los estudiantes, se valoran las potencialidades y necesidades de los mismos y se debate acerca de cuáles son los aspectos significativos a potenciar desde el PEA de la Química orgánica.

Propuesta de actividades docente para potenciar la formación profesional y su vínculo con el desarrollo social.

\section{Actividad \# 1}

Título: Los aminoácidos. Importancia en el organismo humano.

Objetivo: Explicar la estructura funcional de los aminoácidos y la importancia para la salud.

Contenido: Estructura-propiedad-aplicación de los aminoácidos.

Orientación: La actividad se realiza en el desarrollo del tema al explicar la estructura y características generales que presentan los aminoácidos.

Ejecución

El profesor orienta la conformación de dos subgrupos de estudiantes y reparte pequeñas tarjetas y cada una presenta un fragmento de la composición de los aminoácidos, ejemplo:

Tres tarjetas. 1ra: $\mathrm{COOH}$

2da: R 3ra: $\dot{\mathrm{NH}} 2$

Una vez repartidas las tarjetas en el subgrupo, por cada tarjeta se unen dos estudiantes y el profesor les pide que organicen los fragmentos $-\mathrm{COOH},-\mathrm{R}-, \mathrm{y}-\mathrm{NH} 2$ para conformar la función química de los aminoácidos. De ahí, responder los siguientes incisos:

Represente la fórmula química del aminoácido más sencillo de la serie homóloga Esta sustancia por su estructura tiene comportamiento dual. Explique dicho fenómeno basado en la teoría Bronsted-Lowry la propia estructura de los aminoácidos permite unirse entre sí mediante enlaces peptídicos. Explique las características de este enlace mediante una representación.

¿Qué nombre reciben los polímeros que se forman a partir de 20 aminoácidos unidos entre sí por enlaces peptídicos?

¿Qué importancia tienen los aminoácidos en el organismo?

Evaluación: Se evaluará de forma oral mediante el interés que muestren los estudiantes para promover cambios de comportamientos respecto a la responsabilidad en hábitos para proteger la salud.

\section{Actividad \# 2}

Título: Aminoácidos presentes en las proteínas naturales del organismo Objetivo: Nombrar y formular aminoácidos presentes en la composición química de las proteínas y revelar la importancia para la salud. 


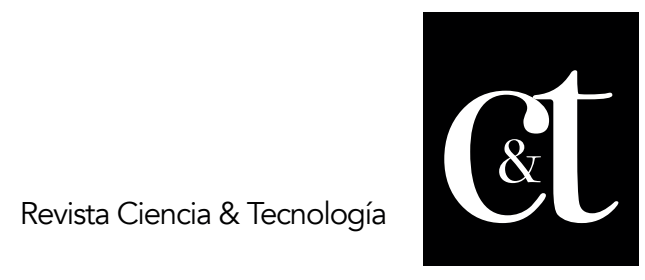

No. 26, 30 de abril de 2020

ISSN impreso: 1390 - 6321

ISSN online: 2661 - 6734

Contenido: Nomenclatura y notación química de los aminoácidos.

Orientación: La actividad se orienta en el desarrollo del tema después de explicar las reglas de nomenclatura y notación química de los aminoácidos.

Ejecución: El profesor revisa el cumplimiento de la actividad orientada en la clase anterior referida a la revisión de la tabla de los 20 aminoácidos presentes en la composición química de las proteínas que se encuentra en la Plataforma Moodle y en la Wikipedia.

A partir de esta información, completa los espacios en blanco:

La glicina se representa en abreviatura como: su nombre científico

Es el ácido metilamina y su fórmulaquímica se representa:

Ala, es la representación abreviada del aminoácido conocido comúnmente como

Se caracteriza por tener una ramificación del grupo metilo y en su cadena principal contiene un solo átomo de carbono, de ahí que su fórmula química se representa:

La notación, Ala--- Val--- Phe, representa una proteína que se forma mediante los enlaces peptídicos de aminoácidos, nombre a cada uno de los aminoácidos que les dieron orígenes:

Evaluación: Se evaluará de forma escrita mediante la revisión de libretas valorando el nivel de preparación del estudio independiente realizado al consultar la plataforma Moodle y el desarrollo de las habilidades en nombrar y formular compuestos orgánicos y además el desarrollo de las habilidades investigativas.

\section{Actividad \#3}

Título: Síntesis de la insulina en el organismo humano.

Objetivo: Explicar las relaciones que se establecen entre la estructura-propiedadaplicaciones de los compuestos orgánicos (aminoácidos).

Contenido: Estructura, propiedades físicas y químicas, y aplicaciones de los aminoácidos

Orientación: La actividad se orienta en clases prácticas dedicadas a resumen del tema aminoácidos.

Ejecución: El profesor comprueba la realización de la búsqueda de información por parte de los estudiantes al estudiar el material de consulta La insulina como hormona. Para la realización exitosa de la actividad, es necesaria la previa preparación.

Dada la siguiente información responda:

La insulina es una hormona producida por el páncreas, con un peso molecular de alrededor de 6000. Contiene dos cadenas peptídicas. La cadena A presenta 21 aminoácidos y la B 30 aminoácidos. Ambas cadenas están unidas por dos puentes disulfuro.

¿Cuál es la función de esta hormona a nivel celular?

Se conoce que es sintetizada inicialmente como única cadena preproinsulina, luego pierde un péptido terminal y se transforma en proinsulina en el retículo endoplasmático. Explique su estructura mediante el tipo de enlace que se presenta entre los aminoácidos 


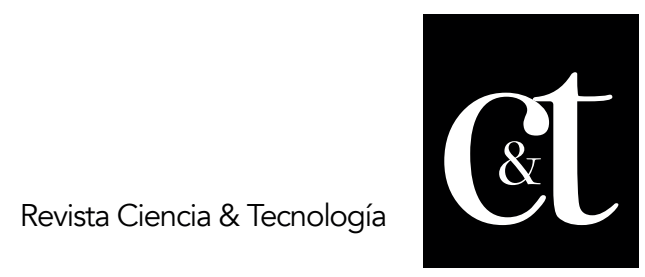

No. 26, 30 de abril de 2020

ISSN impreso: 1390 - 6321

ISSN online: 2661 - 6734

que la conforman.

Debido al valor de la masa molar de esta proteína explique los valores de T.e y T.f. Explique qué trastornos puede ocasionar la producción en exceso o déficits de insulina en el organismo.

Mencione algunas medidas pueden asumirse para mantener los parámetros idóneos en la síntesis de esta sustancia para mantener una salud sana.

Evaluación: Se evaluará de forma escrita mediante la revisión de libretas valorando el nivel de preparación del estudio independiente realizado al consultar la plataforma Moodle y el desarrollo de las habilidades investigativas.

\section{Actividad \#4}

Título: El interferón: una proteína natural y sintética eficaz para el tratamiento de diversas enfermedades.

Objetivo: Explicar las relaciones que se establecen entre la estructura-propiedadaplicaciones de los compuestos orgánicos (aminoácidos).

Contenido: Estructura, propiedades físicas y químicas, y aplicaciones de los aminoácidos

Orientación: La actividad se orienta en el seminario de los aminoácidos

Ejecución: El profesor comprueba la realización de la búsqueda de información por parte de los estudiantes al estudiar el material de consulta " Interferón y su función en el organismo" cómo previa preparación para la realización exitosa de la actividad. Se orienta completar los espacios en blancos de la actividad basada en la correspondencia las aplicaciones de las sustancias dependen de sus propiedades y estas a su vez de sus estructuras:

a) El interferón, es un medicamento de eficaz tratamiento en la afección de diversas enfermedades; entre ellas la mielofribrosis. Su estructura presenta aminoácidos unidos entre sí por enlaces_. Uno de los residuos de aminoácidos presentes en la molécula puede representarse se corresponde con el aminoácido

por su peso molecular presenta t.e. Este medicamento es capaz de evitar la replicación de células malignas, por lo que actúa como mielofribrosis se orientan las siguientes medidas:

1.-

2.-

3.-

Evaluación: Se evaluará de forma oral, para valorar la adquisición de conocimientos relacionados a la estructura-propiedades y aplicaciones de las sustancias. Además, se considera el desarrollo de hábitos y habilidades, así como la conducta en el debate acerca de la prevención de enfermedades.

\section{Actividad \#5}

Título: Enfermedad del déficit de vit B12: Beri-Beri.

Objetivo: Explicar las relaciones que se establecen entre la estructura-propiedadaplicaciones de los compuestos orgánicos (aminoácidos). 


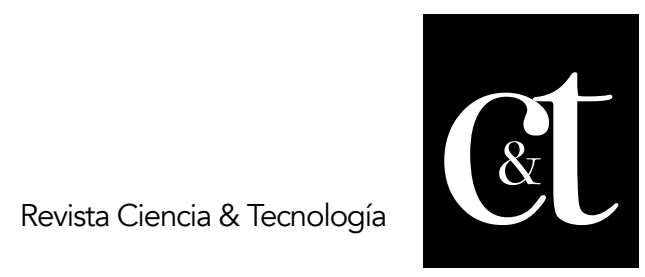

No. 26, 30 de abril de 2020

ISSN impreso: 1390 - 6321

ISSN online: 2661 - 6734

Contenido: Estructura, propiedades físicas y químicas, y aplicaciones de los aminoácidos

Orientación: La actividad se orienta en clase práctica de los aminoácidos Ejecución: El profesor explica en que consiste la técnica Yo soy... y la aplica de la manera siguiente: le ofrece a cada estudiante una tarjeta que presenta el nombre o una fórmula de un residuo de aminoácido. Luego se realiza una auto preparación de los mismos utilizando materiales de consulta que pone el profesor a disposición de los grupos. Así, los estudiantes deben explicar la estructura que presentan los aminoácidos que les dieron origen a esos residuos.

Además, deben exponer las conclusiones arribadas acerca de los siguientes aspectos:

Estructura química de la vitamina B12

Enlaces que se establecen entre los aminoácidos presentes

Síntomas que se manifiestan ante la enfermedad Beri-Beri

Causas que provocan la enfermedad.

Alimentos ricos en Vit B12 a tener en cuenta en la dieta para evitar dicha afección.

Evaluación: Se evaluará de forma oral mediante la exposición y el desarrollo de habilidades profesionales.

\section{Actividad \#6}

Título: El Marasmo y wachascol; enfermedades por déficit de proteínas. Objetivo: Explicar las relaciones que se establecen entre la estructura-propiedad- aplicaciones de los compuestos orgánicos (aminoácidos).

Contenido: Estructura, propiedades físicas y químicas, y aplicaciones de los aminoácidos Orientación: La actividad se realiza en la clase que concluye el tema aminoácidos, puesto que fue orientada con 15 días de antelación.

Ejecución: El profesor comprueba la realización de la búsqueda de información por parte de los estudiantes al estudiar el material de consulta "enfermedades transmisibles y no transmisibles" Los estudiantes deben debatir sus resultados en el grupo y promover actitudes responsables respecto a elevar la calidad de vida. Para ello los estudiantes, se guiaron por los siguientes aspectos:

- Relación de los aminoácidos con la nutrición,

- Causas de estas enfermedades,

- Medidas de prevención,

- Evaluación: Se evaluará de forma oral y escrita mediante la exposición y el desarrollo de habilidades profesionales.

\section{Conclusiones}

Considerar la naturaleza social de la ciencia, los intereses económicos y políticos que la condicionan, así como las implicaciones éticas conduce a desarrollar importantes actitudes y valores en los estudiantes desde las experiencias diarias y las necesidades de asumir un rol protagonista en la calidad de vida individual y colectiva por la influencia de la educación científica que reciben los estudiantes. La enseñanza de la Química orgánica enfrenta realidades en cuanto a intereses individuales y colectivos en relación al conocimiento científico y su repercusión social. Las tareas docentes permitieron constatar el interés de los estudiantes por aprender los contenidos del programa con las 


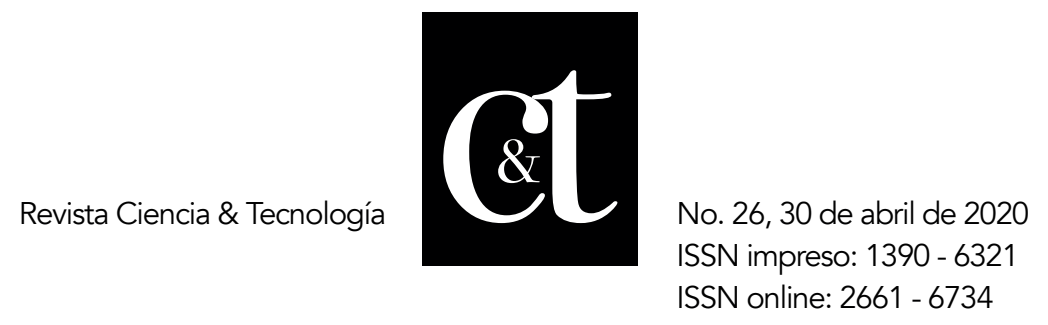

interrogantes necesarias para aplicarlos a la vida diaria.

\section{Referencias bibliográficas}

Addine, F. et al. Didáctica, teoría y práctica. La Habana: Editorial Pueblo y Educación, 2004. p. 309.

Álvarez, A. (2007). La educación como base del desarrollo del ser humano: modelo centrado en el aprendizaje. Educere v.11 n.36 supl.36 Meridad mar. pp. 47-52. Recuperado de http://ve.scielo.org/pdf/edu/v11n36/art07.pdf

Blanco, A. (2003). Fundamentos filosóficos de la educación. La Habana: Pueblo y Educación.

Carvajal, C. (Junio de 2000). Un enfoque para interpretar la relación entre algunas categorías pedagógicas y conceptos relacionados con la salud en el proceso pedagógico. La Habana: Educación CTS.

Delgado, C. (2008). Bioética y política. Santiago: Editorial Universitaria de la República de Cuba, $\mathrm{n}^{\circ} .117$.

. (2009). Una aproximación «no» ¿científica? al tema de los alimentos transgénicos y el maíz FR-Bt1. La Habana: Acuario. 